\title{
Evolution of pathogen virulence under selective predation : A construction method to find eco-evolutionary cycles
}

\author{
Kisdi, Eva
}

2013-12-21

Kisdi , E , Geritz , S A H \& Boldin , B 2013 , ' Evolution of pathogen virulence under selective predation : A construction method to find eco-evolutionary cycles ' , Journal of Theoretical Biology , vol. 339 , pp. 140-150 . https://doi.org/10.1016/j.jtbi.2013.05.023

http://hdl.handle.net/10138/173656

https://doi.org/10.1016/j.jtbi.2013.05.023

acceptedVersion

Downloaded from Helda, University of Helsinki institutional repository.

This is an electronic reprint of the original article.

This reprint may differ from the original in pagination and typographic detail.

Please cite the original version. 


\title{
Evolution of pathogen virulence under selective predation: A construction method to find eco-evolutionary cycles*
}

\author{
Éva Kisdi ${ }^{\dagger}$, Stefan A. H. Geritz ${ }^{1}$, and Barbara Boldin ${ }^{2}$ \\ ${ }^{1}$ Department of Mathematics and Statistics, University of Helsinki, \\ PO Box 68, 00014 Helsinki, Finland \\ ${ }^{2}$ Faculty of Mathematics, Natural Sciences and Information Technologies, \\ University of Primorska, Glagoljaška 8, SI-6000 Koper, Slovenia
}

This is the Author Version of an article published in the Journal of Theoretical Biology: http://dx.doi.org/10.1016/j.jtbi.2013.05.023

\section{Please cite as:}

Kisdi E., S. A. H. Geritz \& B. Boldin. 2013. Evolution of pathogen virulence under selective predation: A construction method to find eco-evolutionary cycles. J. theor. Biol. 339: 140-150.

Creative Commons CC BY NC ND license

\begin{abstract}
We investigate eco-evolutionary cycles in the joint dynamics of pathogen virulence and predator population density when hosts carrying virulent infections are exposed to increased risk of predation. We introduce a new technique to find trade-off functions under which the model exhibits limit cycles; this technique provides a constructive proof that the system is able to generate limit cycles, and can be applied to other eco-evolutionary models as well. We also study a concrete example to confirm that eco-evolutionary cycles occur in a significant part of the parameter space and to briefly explore other evolutionary outcomes in the same model.
\end{abstract}

Keywords: Bogdanov-Takens bifurcation; limit cycle; nonequilibrium dynamics; selective predation; Hopf bifurcation; trade-off; virulence evolution

\footnotetext{
${ }^{*}$ This paper is dedicated to Peter Abrams with reverence for his scientific legacy. S.G. and E.K. are honoured by his personal acquaintance and support.

${ }^{\dagger}$ Corresponding author. email: eva.kisdi@helsinki,fi
} 


\section{Introduction}

In nature, diseased animals do not die on their deathbed. Rather, they are often preferred victims of predators, who selectively prey upon the weak (Hudson et al. 1992; Murray et al. 1997; Johnson et al. 2006; Otti et al. 2012); also more generally, diseased individuals may suffer extra mortality from their disadvantaged position in ecological interactions. The ecological embedding of the host is essential to understand the dynamics of infectious diseases in natural populations, and, conversely, the presence of a disease can change the dynamics of interacting species (Anderson and May 1986; Venturino 2001, 2002, 2007; Packer et al. 2003; Hethcote et al. 2004; Hall et al. 2005; Hilker and Schmitz 2008; Roy and Holt 2008; Kooi et al. 2011; Gyllenberg et al. 2012; Roberts and Heesterbeek 2013; see Hatcher et al. 2006 for a review).

Many models have addressed the evolution of pathogens assuming that infected hosts die at a constant rate determined by the virulence of the pathogen. This assumption is however not valid when mortality derives from ecological interactions and hence depends on the abundance of conspecifics or of other species, such as predators (Williams and Day 2001; Donnelly et al. 2012). For example, infected individuals who might recover (or simply live and reproduce with the infection) in a predator-free environment may be at considerably higher risk when predators are abundant and prefer prey weakened by the infection. Ecological feedback in the disease dynamics can alter the evolution of pathogens fundamentally; it can reverse how the pathogen evolves in response to changing host mortality (Choo et al. 2003), and it can facilitate the coexistence of different pathogen strains (Andreasen and Pugliese 1995, Svennungsen and Kisdi 2009, Morozov and Best 2012). The role of ecological interactions of the host in the evolution of pathogens has been conspicuously neglected until very recently (Choo et al. 2003; Morozov and Adamson 2011; Hoyle et al. 2012; Morozov and Best 2012).

In this paper, we investigate the eco-evolutionary dynamics of a pathogen when its host is subject to predation. We start with a simple host-pathogen model where the densities of susceptible and infected hosts settle at an equilibrium, and the virulence of the pathogen evolves to an optimum where $R_{0}$, the basic reproduction number of the disease, is maximized under a trade-off between transmission and virulence (Bremermann and Thieme 1989). We then introduce a predator that preferentially preys upon hosts infected with virulent pathogens. Because pathogens can evolve on the timescale of years (Fenner 1983), the evolutionary timescale of the pathogen is often similar to the ecological timescale of the predator. We thus assume that pathogen virulence and predator density change on the same timescale, whereas we simplify the mathematical analysis by assuming that the infection-recovery dynamics and the population dynamics of the host are fast. We investigate the eco-evolutionary dynamics of the predator and of virulence, with particular focus on the possibility of long-term nonequilibrium behaviour (limit cycles).

The aim of this paper is twofold. First, we introduce a technique to investigate whether the eco-evolutionary model has the potential to generate limit cycles. In most evolutionary models, an essential element is the choice of trade-off functions that link the evolving traits to each other (in our model, link virulence to transmission and to the rate of predation). Rather than choosing specific trade-off functions a priori, we ask whether there exist possible (and biologically plausible) trade-off functions that lead to periodic dynamics. This idea is inspired by critical function analysis and related techniques (de Mazancourt and Dieckmann 2004; Bowers et al. 2005; Kisdi 2006; Geritz et al. 
2007; Kisdi and Boldin 2013), but the conditions required for cycles are somewhat more involved. Specifically, in the first part of the paper we show that the trade-off functions can be chosen such that the system has a supercritical Bogdanov-Takens bifurcation. The Bogdanov-Takens point is a common point of a Hopf bifurcation line with saddle-node and homoclinic bifurcation lines (Kuznetsov 2004, p. 324; see Table 1 for an overview of bifurcations encountered in this paper). The existence of the Bogdanov-Takens point thus guarantees the existence of a Hopf bifurcation, and since the Hopf bifurcation is shown to be supercritical, there must be a region in parameter space where the model exhibits stable limit cycles of pathogen virulence and predator density.

In the second part of the paper, we follow traditional methods to analyse a concrete example where we assume a fixed function for the transmission-virulence trade-off and link the predator's capture rate to virulence via a parameterised family of functions. Using the parameters of the latter function as bifurcation parameters, we explore how the shape of the trade-off influences the joint dynamics of predators and virulence. This analysis confirms that eco-evolutionary cycles can occur in a significant part of the parameter space. We briefly consider also whether virulence is under stabilizing or disruptive selection when it arrives at an equilibrium (disruptive selection leads to evolutionary branching if virulence evolution is slower than predator dynamics; see Morozov and Best 2012) and how an established host-pathogen system responds to a newly introduced predator. In the Discussion, we compare our model to eco-evolutionary cycles in predator-prey systems (see Abrams 2000b for a review).

Table 1. Overview of bifurcations encountered in this paper

\begin{tabular}{|c|c|c|}
\hline Bifurcation & Description & Condition $^{1}$ \\
\hline saddle-node & two equilibria collide and disappear & $\operatorname{Det} \mathbf{J}=0$ \\
\hline $\begin{array}{l}\text { Hopf } \\
\text { supercritical }\end{array}$ & $\begin{array}{l}\text { a limit cycle appears around an equilibrium } \\
\text { a stable limit cycle appears when the equilib- } \\
\text { rium becomes unstable }\end{array}$ & $\operatorname{Tr} \mathbf{J}=0, \operatorname{Det} \mathbf{J}>0$ \\
\hline subcritical & $\begin{array}{l}\text { an unstable limit cycle appears when the equi- } \\
\text { librium becomes stable }\end{array}$ & \\
\hline Bogdanov-Takens & $\begin{array}{l}\text { common point of a saddle-node and a Hopf } \\
\text { bifurcation line, a homoclinic bifurcation line } \\
\text { must end here }\end{array}$ & $\operatorname{Tr} \mathbf{J}=0, \operatorname{Det} \mathbf{J}=0$ \\
\hline homoclinic & $\begin{array}{l}\text { a limit cycle collides with a saddle and disap- } \\
\text { pears }\end{array}$ & global bifurcation \\
\hline transcritical & $\begin{array}{l}\text { two equilibria cross and exchange stability; } \\
\text { typically one equilibrium is on the boundary, } \\
\text { which corresponds to the absence of a species, } \\
\text { and the other equilibrium moves across as it } \\
\text { enters the positive quadrant }\end{array}$ & $\begin{array}{l}\text { the diagonal element of } \\
\mathbf{J} \text { corresponding to the } \\
\text { absent species is zero }\end{array}$ \\
\hline cusp & $\begin{array}{l}\text { common point of two saddle-node bifurcation } \\
\text { lines }\end{array}$ & $\begin{array}{l}\text { involves higher deriva- } \\
\text { tives }\end{array}$ \\
\hline
\end{tabular}

${ }^{1}$ given for 2-dimensional systems and omitting non-degeneracy conditions.

$\mathbf{J}$ is the Jacobian matrix. 


\section{The model}

We consider a host-pathogen-predator system with evolving pathogens. For simplicity, we use a single-infection SIS-model, where we assume that pathogens spread only by direct horizontal transmission, the host population is regulated by density-dependent birth, incidence follows the mass action law and the predator has a linear functional response. Pathogens with higher virulence have higher transmission rates, but also impose higher predation risk on infected hosts. Mutations of the pathogen maintain a narrow distribution of virulence such that virulence evolves proportionally to the gradient of between-host selection (Abrams et al. 1993a; Day and Proulx 2004). These assumptions lead to the equations

$$
\begin{aligned}
\frac{d S}{d t} & =r b(N) N-\mu S-c P S-\beta(\alpha) S I+\nu I \\
\frac{d I}{d t} & =[\beta(\alpha) S-(\mu+k \alpha+\nu+(c+f(\alpha)) P)] I \\
\frac{d P}{d t} & =[\gamma(c N+f(\alpha) I)-d] P \\
\frac{d \alpha}{d t} & =v\left[\beta^{\prime}(\alpha) S-k-f^{\prime}(\alpha) P\right]
\end{aligned}
$$

where $S, I, P$, and $\alpha$ denote respectively the density of susceptible hosts, infected hosts, predators, and the pathogen's virulence, and $N=S+I$ is the total host density. All hosts give birth to susceptible offspring at the rate $r b(N)$, where $r$ is the intrinsic birth rate and $b$ is a decreasing function with $b(0)=1$ and $\lim _{N \rightarrow \infty} b(N)<\mu / r . \mu, \nu$ and $c$ denote respectively the background death rate of hosts, the recovery rate and the rate at which predators capture healthy hosts. $\beta$, the transmission rate, is an increasing function of virulence. The infection increases the host's death rate by $k \alpha$ and also increases the predator's capture rate by $f(\alpha)$. We introduce the factor $k$ into the rate of disease-induced mortality such that we can study the effect of direct mortality relative to predation risk. $k=0$ corresponds to a disease that is not lethal per se, yet weakens the host such that it is more prone to predation. $f(\alpha)$ is an increasing function of virulence so that more virulent infections make it easier for the predator to catch the host, and the disease does not affect the appeal or nutritional value of the host. The predator cannot contract the disease. The predators convert the captured hosts into predator offspring with efficiency $\gamma$, and die at a constant rate $d$.

To derive equation $(1 \mathrm{~d})$, note that a rare mutant strain with virulence $\alpha_{m u t}$ spreads according to

$$
\frac{d I_{m u t}}{d t}=\left[\beta\left(\alpha_{m u t}\right) S-\left(\mu+k \alpha_{m u t}+\nu+\left(c+f\left(\alpha_{m u t}\right)\right) P\right)\right] I_{m u t}
$$

The selection gradient is the derivative of the mutant growth rate, given by the expression in the brackets in (2), with respect to the mutant trait value $\alpha_{m u t}$, evaluated at $\alpha_{m u t}=\alpha$. This selection gradient appears in the right hand side of (1d) multiplied with the speed constant $v$.

To facilitate the analysis, we make two simplifying assumptions concerning the time scales involved in the dynamics in (1). Firstly, we assume that the host population dynamics is fast relative to the predator dynamics and to the evolution of virulence (i.e., $\gamma, d$ and $v$ are small). This 
allows us to consider the planar system of (1c)-(1d) in isolation, assuming that $S$ and $I$ are at the quasi-equilibrium values of the fast system (1a)-(1b). Secondly, we assume $r \rightarrow \infty$. This implies that the total population size of the host, $N=S+I$, instantly equilibrates to the value given implicitly by $b(N)=0 . N$ is therefore a given constant, which greatly simplifies the dynamics of the fast time scale. From (1b), the host dynamics has the quasi-equilibrium

$$
\begin{aligned}
S & =\frac{\mu+k \alpha+\nu+(c+f(\alpha)) P}{\beta(\alpha)} \\
I & =N-S
\end{aligned}
$$

and it is easy to see that this quasi-equilibrium is always stable. Biologically, the assumption $r \rightarrow \infty$ means that each time a host dies, it is instantly substituted by a newborn such that the total size of the host population is unchanged; but because newborns are susceptible, host death still affects the ratio of healthy and diseased hosts. When assuming $r \rightarrow \infty$, we must also assume that $\gamma c N<d$ such that the predator cannot maintain its population solely on susceptible hosts; this assumption is necessary because otherwise the instant birth of susceptible hosts would fuel unlimited growth of the predator. Our time-scale separation assumptions are motivated by tractability, yet may be acceptable approximations for example for insect hosts and their mammalian predators, as insects can have very high fecundities and much shorter lifespan than small mammals. At the end of this paper, we demonstrate by a numerical example that our simplifying time-scale assumptions are not necessary for the eco-evolutionary cycles we find analytically below.

\section{Eco-evolutionary cycles of predator density and pathogen viru- lence}

We are interested in the dynamics of the slow system (1c)-(1d) with $S$ and $I$ substituted from (3),

$$
\begin{aligned}
\frac{d P}{d t} & =[\gamma(c N+f(\alpha)(N-S(P, \alpha)))-d] P \\
\frac{d \alpha}{d t} & =v\left[\beta^{\prime}(\alpha) S(P, \alpha)-k-f^{\prime}(\alpha) P\right] \\
S(P, \alpha) & =\frac{\mu+k \alpha+\nu+(c+f(\alpha)) P}{\beta(\alpha)}
\end{aligned}
$$

where $N$ is a constant. Notice that because $S(P, \alpha)$ is linear in $P$ and $\frac{1}{P} \frac{d P}{d t}$ is linear in $S(P, \alpha)$, there is only one nonzero equilibrium of $P$ for any fixed value of $\alpha$. The right hand sides of both differential equations are however nonlinear in $\alpha$, such that depending on the unspecified functions $\beta$ and $f$, the system may have multiple equilibria. Notice also that at each equilibrium, we have

$$
\frac{\partial S(P, \alpha)}{\partial \alpha}=\frac{k+f^{\prime}(\alpha) P}{\beta(\alpha)}-S(P, \alpha) \frac{\beta^{\prime}(\alpha)}{\beta(\alpha)}=-\frac{1}{\beta(\alpha)} \frac{d \alpha}{d t}=0
$$

The Jacobian of (4) evaluated at an interior equilibrium is given by

$$
\mathbf{J}=\left(\begin{array}{ll}
J_{11} & J_{12} \\
J_{21} & J_{22}
\end{array}\right)
$$


with

$$
\begin{aligned}
& J_{11}=-\gamma f(\alpha)(c+f(\alpha)) P / \beta(\alpha) \\
& J_{12}=\gamma(N-S(P, \alpha)) f^{\prime}(\alpha) P \\
& J_{21}=v\left[(c+f(\alpha)) \beta^{\prime}(\alpha) / \beta(\alpha)-f^{\prime}(\alpha)\right] \\
& J_{22}=v\left[\beta^{\prime \prime}(\alpha) S(P, \alpha)-f^{\prime \prime}(\alpha) P\right]
\end{aligned}
$$

where (5) was used to simplify $J_{12}$ and $J_{22}$.

\subsection{Construction method}

In order to show that the model is able to produce cycles of predator density and pathogen virulence, we show that the trade-off functions $\beta$ and $f$ can be chosen such that the equilibrium undergoes a supercritical Hopf bifurcation, which gives rise to a stable limit cycle (see Table 1 for a brief explanation of bifurcations encountered in this paper). A brief outline of the method is summarized as follows. First, we choose values for the evolving traits (virulence, transmission rate, and predation rate) such that the ecological system has a biologically feasible equilibrium, i.e., all population densities are positive. Second, we choose the slopes of the trade-offs such that the evolutionary dynamics in (4b) has an equilibrium at the chosen trait values. Next, we need to choose the remaining slope and the convexities of the trade-offs such that the Jacobian matrix has a pair of purely imaginary eigenvalues, which implies a Hopf bifurcation. In 2-dimensional systems as in (4), this requires that the trace of the Jacobian is zero whereas the determinant is positive. To ensure the latter, as a third step we choose the slopes and the convexities such that both the trace and the determinant are zero, which (generically) corresponds to a Bogdanov-Takens bifurcation point; and then unfold this into a positive determinant, whereby we obtain a Hopf bifurcation. The last step is to make sure that the Hopf bifurcation is supercritical (note that if the Hopf bifurcation is subcritical, then the system has an unstable limit cycle near the Bogdanov-Takens point and all other orbits are attracted to equilibria). In other words, we obtain the cycles such that we place the system at a Bogdanov-Takens bifurcation point, which is the common point of a Hopf bifurcation line, a saddle-node bifurcation line and a homoclinic bifurcation line (Kuznetsov 2004, p. 324; see figures 2-3 below), and then unfold this degeneracy by using the Taylor coefficients (slope and convexity) of the trade-offs as bifurcation parameters. If the Hopf bifurcation is supercritical, then the system exhibits a stable limit cycle inbetween the Hopf and homoclinic bifurcation lines.

In the remainder of this section, we carry out the above construction method. At the BogdanovTakens bifurcation point, four equations must be satisfied simultaneously, two for the equilibrium conditions and two for the trace and the determinant of the Jacobian to be zero: (i) $\frac{1}{P} \frac{d P}{d t}=0$; (ii) $\frac{d \alpha}{d t}=0$; (iii) $J_{11}+J_{22}=0$; and (iv) $J_{11} J_{22}-J_{12} J_{21}=0$. Fix all parameter values but leave the functions $\beta$ and $f$ as yet unspecified. Choose positive values for $\alpha, \beta(\alpha)$ and $f(\alpha)$ such that equation (i) yields a positive equilibrium value for $P$ that satisfies $N-S(P, \alpha)>0$. [Note that " $\beta(\alpha)$ " and " $f(\alpha)$ " denote the specific values the functions assume at a given value of $\alpha$; the functions themselves are not yet specified except at this one point.] With $\alpha, \beta(\alpha)$ and $f(\alpha)$ given and $P$ obtained from the linear equation (i), $J_{11}$ evaluates to a number. From (iii) we have $J_{22}=-J_{11}$, such that (iv) can be written as (iv*) $J_{12} J_{21}=-J_{11}^{2}$. Substituting $\beta^{\prime}(\alpha)=\left(k+f^{\prime}(\alpha)\right) / S(P, \alpha)$ from equation (ii) 
into $J_{21}$ makes (iv*) a quadratic equation for $f^{\prime}(\alpha)$,

$$
\frac{v \gamma(N-S(P, \alpha)) P}{\beta(\alpha) S(P, \alpha)} f^{\prime}(\alpha)\left[k(c+f(\alpha))-(\beta(\alpha) S(P, \alpha)-(c+f(\alpha)) P) f^{\prime}(\alpha)\right]=-J_{11}^{2}
$$

where the coefficient of the $\left[f^{\prime}(\alpha)\right]^{2}$ term is negative since by $(4 \mathrm{c}),(\beta(\alpha) S(P, \alpha)-(c+f(\alpha)) P)=$ $\mu+k \alpha+\nu>0$. Further, the left hand side of $(7)$ is zero at $f^{\prime}(\alpha)=0$ and the right hand side is negative. Taken together, these properties imply that the equation has exactly one positive and one negative solution for $f^{\prime}(\alpha)$. Biologically, $f$ and $\beta$ are expected to be increasing functions of virulence, hence choose the positive root of (7) and the corresponding positive $\beta^{\prime}(\alpha)=\left(k+f^{\prime}(\alpha)\right) / S(P, \alpha)$ from (ii) for the first-order Taylor coefficients of $f$ and $\beta$, respectively. With these slopes of the trade-off functions, equations (i), (ii) and (iv*) are satisfied. Finally, choose $f^{\prime \prime}(\alpha)$ and $\beta^{\prime \prime}(\alpha)$ such that they satisfy (iii), i.e., let $\beta^{\prime \prime}(\alpha)=\frac{1}{S(P, \alpha)}\left[f^{\prime \prime}(\alpha) P-J_{11} / v\right]$.

If the values of $\beta^{\prime}(\alpha), \beta^{\prime \prime}(\alpha)$ and $f^{\prime}(\alpha)$ are as specified above, then the chosen values of $\alpha, \beta(\alpha)$ and $f(\alpha)$ represent an equilibrium of (4) with two zero eigenvalues of the Jacobian. The above construction leaves $f^{\prime \prime}(\alpha)$ as yet unspecified, but does not yet guarantee that the bifurcation is a generic Bogdanov-Takens bifurcation and that it is associated with a supercritical Hopf bifurcation. In Appendix A, we show that $f^{\prime \prime}(\alpha)$ can be chosen such that this last criterion is satisfied.

As shown above, the critical slopes of the trade-offs are positive, which is biologically realistic, because both transmission and predation risk are expected to increase with virulence. The convexities can be chosen with somewhat more freedom because $f^{\prime \prime}(\alpha)$ needs to satisfy only an inequality (see Appendix A). Properties other than the first and second derivatives of the trade-off functions at the chosen trait values are irrelevant for obtaining the limit cycle, hence the trade-off functions can be extended in any biologically meaningful way over the whole domain of $\alpha$. The BogdanovTakens point therefore corresponds to a biologically plausible system, and, by continuity, also limit cycles are obtained with plausible trade-off functions.

\subsection{The mechanism driving the cycles}

The key mechanism driving every cycle we find in this study is that high predator density selects for decreasing pathogen virulence. This is possible because the predator's capture rate towards the infected hosts increases with the virulence of the infecting strain, which means that the pathogen can avoid excess predation by evolving lower virulence. If the predator killed the infected hosts independently of pathogen virulence (i.e., if $f$ were constant), then predation would be akin to an increase in the background mortality rate, and therefore would always select the pathogen for faster transmission at the cost of higher virulence (Lenski and May 1994).

As the pathogen evolves low virulence due to the selective pressure from high predator density, the predator's capture rate decreases, which implies that predator density starts to decline. This diminishes selection for low virulence, and therefore virulence will increase again in order to increase the transmission rate of the pathogen. Increasing virulence increases the predator's capture rate, and, in turn, its density, whereby the eco-evolutionary cycle closes. 
There is, however, an alternative scenario that could also explain the cycles. Suppose, for the sake of the argument, that high predator density selects for increasing virulence in the first place, as it does when $f$ is constant. Higher virulence implies a shorter lifespan of the infected and a decline of infected hosts. Since this means fewer easy prey, predator density decreases, which diminishes the selection pressure on the pathogen. Under our current hypothesis virulence evolves lower again; and as the density of infected hosts increases, the predator returns to its initial high density. This alternative scenario predicts cycles in the opposite direction (anticlockwise vs clockwise) relative to the first mechanism.

To see which of the above alternatives drives the cycles found in this model, notice that increasing predator density $P$ while keeping $\alpha$ fixed changes the selection gradient on virulence in two distinct ways: increasing $P$ decreases the selection gradient via the negative term $-f^{\prime}(\alpha) P$ in (4b) but increases the positive term $\beta^{\prime}(\alpha) S(P, \alpha)$ via increasing $S(P, \alpha)$ (cf. (4c)). Both effects are linear in $P$ and the negative effect dominates if

$$
f^{\prime}(\alpha)>\beta^{\prime}(\alpha) \frac{c+f(\alpha)}{\beta(\alpha)}
$$

Suppose that a limit cycle goes through the points $\left(P_{1}, \alpha\right)$ and $\left(P_{2}, \alpha\right)$ where the above inequality holds, and let $P_{1}<P_{2}$. The selection gradient on virulence must then be positive at $P_{1}$ but negative at $P_{2}$; hence virulence decreases when predator density is high, and the cycle operates according to the first scenario described above.

In the remainder of this section, we show that any limit cycle born via a Hopf bifurcation must satisfy condition (8). For a Hopf bifurcation to occur, the trace of the Jacobian must be zero whereas the determinant must be positive. Since $J_{11}$ is always negative (see (6)), $J_{11}+J_{22}=0$ implies $J_{22}>0$ and therefore $J_{11} J_{22}<0$. The determinant is positive if $J_{12} J_{21}<J_{11} J_{22}<0$. Because $J_{12}$ is non-negative, $J_{21}$ must be negative, which is equivalent to condition (8) evaluated at the bifurcating equilibrium. By continuity, (8) evaluated at the $\alpha$-coordinate of the equilibrium inside the limit cycle continues to hold also in the vicinity of the bifurcation point, and since the direction of a limit cycle cannot change, all limit cycles that form a continuous family with a Hopf bifurcation will have the same property.

The existence of a limit cycle around an equilibrium where the opposite of (8) holds cannot be excluded. Such a limit cycle can not undergo a Hopf bifurcation, but might be born for example via a fold bifurcation of limit cycles. Our numerical experiments based on the following example did however not find any such cycles.

\section{An example}

In this section, we analyze a concrete example where the model exhibits cycles, with Hopf, saddlenode and homoclinic bifurcations organized by a Bogdanov-Takens point. Here we follow the traditional approach of assuming some trade-off functions at the onset and exploring the dynamics in terms of the parameters of the given trade-off functions. We thus assume that the transmissionvirulence trade-off is given by the fixed function

$$
\beta(\alpha)=\frac{\alpha}{0.1+\alpha}
$$


and the predator's excess capture rate of the infected hosts increases with virulence according to the parameterized function

$$
f(\alpha)=\frac{m \alpha}{e^{-l \alpha}+n \alpha} .
$$

As $\alpha$ goes to infinity, $f(\alpha)$ saturates to the value $m / n$. We keep the saturation value $m / n$ constant as we vary the parameters $l>0$ and $n>0$ to obtain various concave and sigmoidal shapes of $f$ (see figure 1). We shall also assume $k>0$ to ensure that virulence remains bounded.

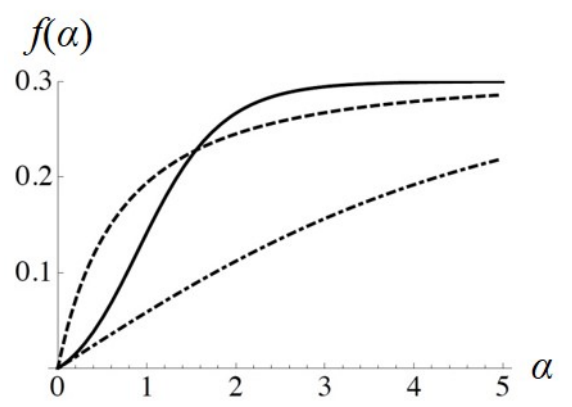

Figure 1: The shape of the function $f$ defined in (9b). $m=0.3 n$ such that each curve is asymptotic to 0.3 . Thick line: $n=0.2, l=1.5$; dashed line: $n=1.5, l=0.2$; dash-dotted line: $n=0.2, l=0.2$.

\subsection{Predator viability and boundary equilibria}

In absence of the predator, the pathogen evolves to its locally optimal virulence where its basic reproduction number $R_{0}(\alpha)=\frac{\beta(\alpha) N}{\mu+k \alpha+\nu}$ is maximal, or, equivalently, the density of susceptible hosts, $S(0, \alpha)$, is minimal (Bremermann and Thieme 1989; Dieckmann and Metz 2006; Metz et al. 2008). The predator-free optimal virulence $\alpha^{*}$ corresponds to the stable equilibrium of equation (4b) with $P=0$. We assume that $\alpha^{*}$ is viable, i.e., $R_{0}\left(\alpha^{*}\right)>1$. The predator, when introduced at a low density, is able to invade the host-pathogen system at $\alpha^{*}$ if $f\left(\alpha^{*}\right)$ is sufficiently high; and with the function in (9b), this holds when $l$ or $n$ is sufficiently large, provided that the saturation value $m / n$ exceeds a threshold (see Appendix B for details). There are no bifurcations of boundary equilibria other than the transcritical bifurcation of the predator-free equilibrium. Note that even if the predator cannot invade the host-pathogen system from low initial density, the full system may still have an attractor with positive predator density and virulence exceeding $\alpha^{*}$.

\subsection{Bifurcations and cycles}

The main panel of figure 2 shows the bifurcation diagram of the model in (4) and (9a,b) with respect to the parameters $l$ and $n$, which control the shape of the function $f$ (cf. figure 1 ). There are two codimension-2 bifurcation points, which organize the bifurcation structure of the model: the Bogdanov-Takens point $B T$ and a cusp point $C$. Inbetween the two saddle-node bifurcation lines $S N$ that emanate from the cusp, the system has three equilibrium points. The phase portraits in figure 2 show the lower two equilibria $\left(P_{1}, \alpha_{1}\right)$ and $\left(P_{2}, \alpha_{2}\right)$; the third equilibrium, $\left(P_{3}, \alpha_{3}\right)$, has the highest coordinates on both axes and in these examples it is too far to be shown conveniently. 
$\left(P_{2}, \alpha_{2}\right)$ is always a saddle, whereas $\left(P_{3}, \alpha_{3}\right)$ is always asymptotically stable. $\left(P_{1}, \alpha_{1}\right)$ and $\left(P_{2}, \alpha_{2}\right)$ bifurcate at the upper saddle-node line, whereas $\left(P_{2}, \alpha_{2}\right)$ and $\left(P_{3}, \alpha_{3}\right)$ bifurcate at the lower $S N$ line. Outside the saddle-node lines the system has only one equilibrium, which is stable.
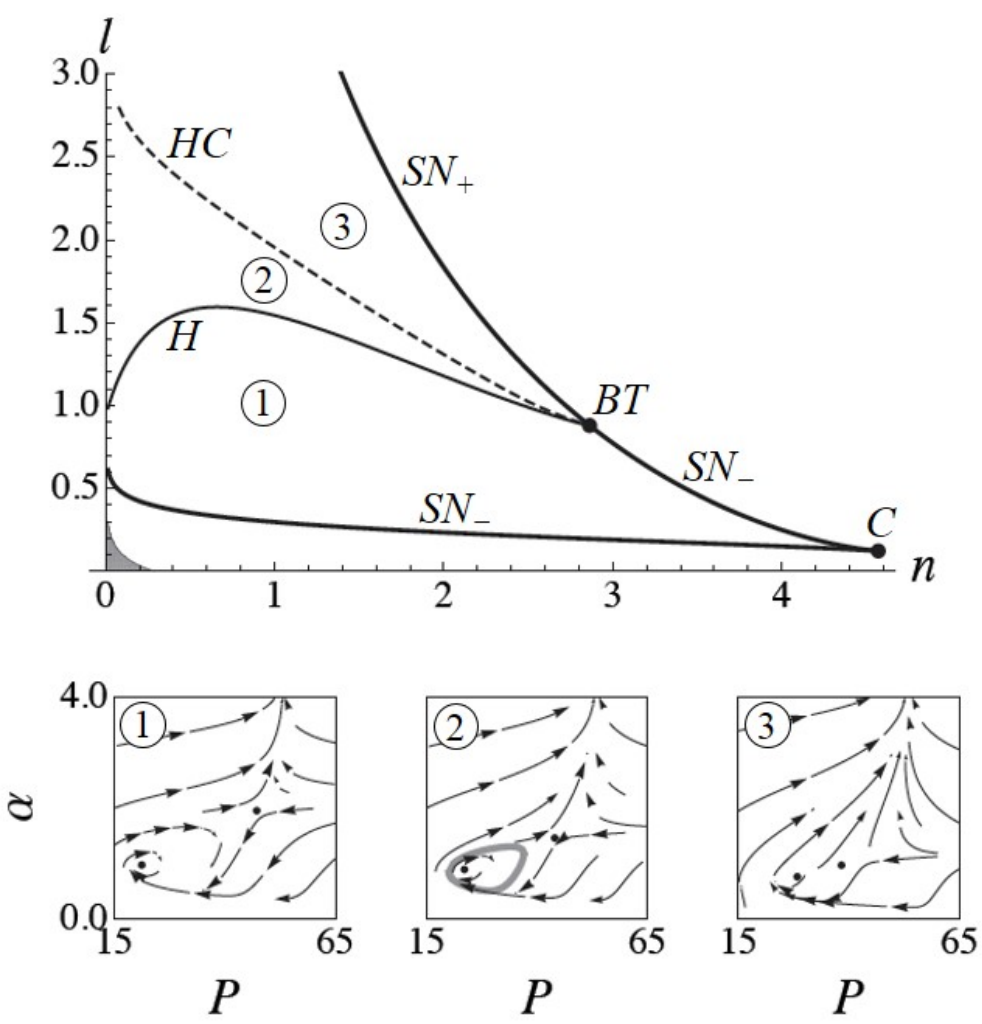

Figure 2: Main panel: bifurcation diagram of the example specified in $(9 \mathrm{a}, \mathrm{b})$. The thick black lines are saddle-node bifurcation lines $\left(S N_{+}, S N_{-}\right)$; the thin line is a Hopf bifurcation line $(H)$; the dashed line is a homoclinic bifurcation line $(H C) . B T$ and $C$ mark the Bogdanov-Takens and cusp points, respectively. In the grey region near the origin, the predator is not viable (i.e., it cannot invade the predator-free equilibrium). The three phase portraits below the main panel show the dynamics near the lower two equilibria $\left(P_{1}, \alpha_{1}\right)$ and $\left(P_{2}, \alpha_{2}\right)$ (dots); the coordinates of the third equilibrium, $\left(P_{3}, \alpha_{3}\right)$, are off the scale, the orbits leaving upwards converge to the third equilibrium. The numbers of the phase portraits correspond to the numbered areas of the bifurcation diagram; $1,\left(P_{1}, \alpha_{1}\right)$ is a stable equilibrium; $2,\left(P_{1}, \alpha_{1}\right)$ is an unstable focus surrounded by a stable limit cycle; 3 , the limit cycle has disappeared via a homoclinic bifurcation. Parameters: $\mu=1, \nu=$ $10, k=0.01, c=0.1, \gamma=0.01, d=0.3, v=0.1, m=0.3 n, N=100$.

In area 1 of the bifurcation diagram, $\left(P_{1}, \alpha_{1}\right)$ is asymptotically stable (either a stable node or, as in the left phase portrait, a stable focus), such that the model has two stable equilibria separated by a saddle. $\left(P_{1}, \alpha_{1}\right)$ loses its stability via a Hopf bifurcation at line $H$, and a stable limit cycle appears in area 2 of the bifurcation diagram (this corresponds to the middle phase portrait in figure 2). The limit cycle grows and eventually collides with the saddle, resulting in a homoclinic structure at the bifurcation line $H C$. Above the homoclinic bifurcation line, i.e., in area 3 of the bifurcation diagram, orbits are attracted to the highest equilibrium $\left(P_{3}, \alpha_{3}\right)$. $\left(P_{1}, \alpha_{1}\right)$ becomes an unstable node that disappears through the upper saddle-node bifurcation line $S N$. Above the Bogdanov-Takens point $B T$, the saddle-node bifurcation involves a saddle and an unstable node 
$\left(S N_{+}\right)$, whereas below the $B T$ point, a saddle and a stable node bifurcate $\left(S N_{-}\right)$.

The system exhibits cycles of predator density and pathogen virulence inbetween the Hopf and homoclinic bifurcation lines (in area 2 of the bifurcation diagram). Since these limit cycles are born via a Hopf bifurcation, they are driven by high predator density selecting for decreasing virulence (see section 3.2); accordingly, the phase portrait of the limit cycle shows clockwise movement. The cycles disappear at high values of $l$ and also at high values of $n$, because in both cases $f$ saturates very quickly and is therefore almost constant. With constant $f$, low virulence does not help the pathogen to avoid predator-related mortality, and therefore high predator density does not select for low virulence, so that the key mechanism driving the cycle is absent.

\subsection{Direct mortality versus predation risk of infected hosts}

In our model, the pathogen increases the mortality of infected hosts as compared to healthy hosts in two ways: it causes direct mortality at rate $k \alpha$, and it increases the predator's capture rate by $f(\alpha)$. We have introduced the factor $k$ so that we can change the direct mortality rate without changing the transmission rate and the capture rate. Increasing the direct mortality rate is expected to diminish the role of the predator in the evolution of virulence. Since the predator plays a key role in the cycles, the cycles may disappear if direct mortality overwhelms the predator-related mortality. Moreover, the predator may go extinct when increasing the direct mortality rate, because the density of the infected hosts decreases and the predator is viable only if there are enough infected hosts (which are the easy prey). Figure 3a shows the bifurcation diagram for a higher value of $k$, i.e., for higher direct mortality caused by the disease. As expected, the eco-evolutionary cycles occur in a smaller region compared to figure 2 , and there is a larger area where the predator is not viable.
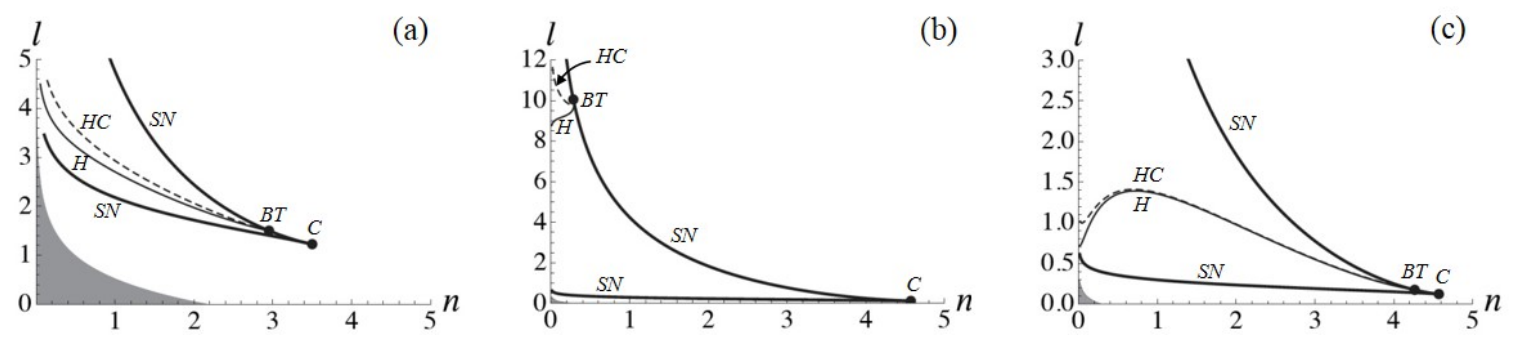

Figure 3: Bifurcation diagrams for (a) $k=0.5$, (b) $v=0.0005$, (c) $v=10$. Notation and other parameters as in figure 2. Notice the different scales on the vertical axis.

\subsection{The relative speed of predator population dynamics and virulence evolution}

Since the eco-evolutionary cycles of this model are caused by the interplay between how the pathogen evolves in response to changes in predator density and how predator density responds to changes in the predator's virulence-dependent capture rate, it is essential for the cycles that predator population density and pathogen virulence change on the same time scale. If either $v \rightarrow 0$ or $v \rightarrow \infty$ such that virulence evolution is either slow or fast compared to the population dynamics 
of the predator, then the planar system in (4) is decomposed into two separate one-dimensional systems with different time scales, which implies that cycles are no longer possible. Instead, the faster variable attains a quasi-equilibrium that tracks the slower variable until the slower variable also reaches its equilibrium.

The value of $v$ does not affect the existence of equilibria of (4), and therefore does not affect the saddle-node bifurcation lines, but it does influence the stability of the equilibria and therefore the Hopf and homoclinic bifurcation lines (compare figure 3b,c with figure 2). As $v$ decreases towards zero, the Bogdanov-Takens point shifts towards the left on the saddle-node bifurcation line (figure $3 \mathrm{~b})$. Since the saddle-node bifurcation line is asymptotic to the vertical axis, the cyclic regime shrinks and eventually vanishes as $v \rightarrow 0$. Increasing $v$ makes the Bogdanov-Takens point move towards the cusp point. In this case, the Hopf and homoclinic bifurcation lines come closer to each other (figure 3c) such that in the limit $v \rightarrow \infty$, the cycles disappear because as soon as the lowest equilibrium $\left(P_{1}, \alpha_{1}\right)$ is destabilized, the orbits are attracted to the stable equilibrium $\left(P_{3}, \alpha_{3}\right)$.

\subsection{Disruptive selection and evolutionary branching of virulence}

At the asymptotically stable equilibria of (4), virulence may be either under stabilizing or under disruptive selection. Selection is disruptive if the mutant growth rate (the expression in the brackets in (2)) has a minimum as a function of $\alpha_{m u t}$, i.e., if

$$
\beta^{\prime \prime}(\alpha) S(P, \alpha)-f^{\prime \prime}(\alpha) P>0
$$

holds at the attractor (Maynard Smith 1982; Abrams et al. 1993b). Disruptive selection occurs at the lower attractor $\left(P_{1}, \alpha_{1}\right)$ in the area delimited from below by the black dashed line in figure 4 (recall that $\left(P_{1}, \alpha_{1}\right)$ loses its asymptotic stability at the Hopf bifurcation line and disappears at the upper saddle-node line, so that these lines delimit the area of disruptive selection from above). At the higher attractor $\left(P_{3}, \alpha_{3}\right)$, selection is disruptive in a tiny area between the lower segment of the black dashed line and the lower saddle-node line (see the inset in figure 4 for details).

If the evolution of virulence is slow compared to all ecological dynamics, i.e., in the case of $v \rightarrow 0$, we arrive at an adaptive dynamic model for the evolution of virulence, where the attracting fitness minima are evolutionary branching points of virulence (Geritz et al. 1998). Since $v$ does not influence the saddle-node bifurcation lines and the stabilizing vs disruptive character of selection, figure 4 is valid also for $v \rightarrow 0$, except that the Hopf and homoclinic bifurcation lines disappear (see above; figure $3 \mathrm{~b}$ ). Evolutionary braching can thus occur in a wide range of parameters at the lower attractor $\left(P_{1}, \alpha_{1}\right)$ in the example shown in figure 4 .

\subsection{Evolutionary response to a new predator}

The question how ecosystems respond evolutionarily to the introduction of new species gains importance because of concerns about invasive species (see e.g. Abrams 2000a, 2012 on modelling evolutionary responses to new species and Strauss et al. 2006 for a review of data). In our model, the presence of the pathogen makes the predator viable, and hence it is natural to ask what happens 


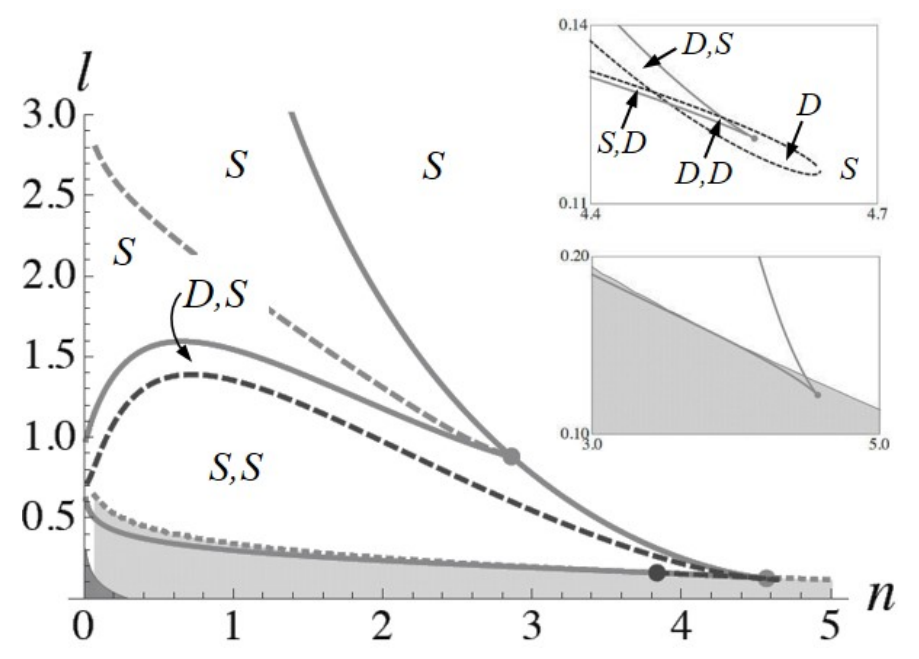

Figure 4: Stabilizing vs disruptive selection and the evolutionary response of pathogens to introducing the predator. Grey lines: bifurcation lines from the main panel of figure 2. The black dashed line separates areas where virulence is under stabilizing $(S)$ versus disruptive $(D)$ selection at the attractor(s) (listed in the order $\left.\left(P_{1}, \alpha_{1}\right),\left(P_{3}, \alpha_{3}\right)\right)$. The upper inset shows this line in the vicinity of the cusp point; in this inset, the saddle-node lines are vertically offset by -0.001 for readability. In the grey area below the grey dotted line, the pathogen responds with evolving lower virulence when the predator is introduced into the host-pathogen system at its predator-free equilibrium; outside this region, higher virulence evolves. The lower inset shows this area in the vicinity of the cusp. Parameters as in figure 2.

when the predator appears anew in an established system of the host and its pathogen. The predator could be introduced also as a measure of biological control, in order to reduce the frequency of infected hosts via selective predation of the infected.

Suppose that the predator is introduced at a low initial density into a host-pathogen system where the pathogen has evolved to its predator-free optimal virulence $\alpha^{*}$ (see section 4.1). Introducing a new predator is similar to the effect of increasing predator density during an eco-evolutionary cycle. Hence if condition (8) holds at $\alpha^{*}$, then the presence of the predator selects for lower virulence at least initially. In figure 4, grey shading indicates the parameter region where the pathogen evolves lower virulence after the introduction of the predator. This grey area covers parts both inside and outside the wedge of the saddle-node lines. Outside the wedge the system with the predator has only one equilibrium, for which $\alpha<\alpha^{*}$ holds in the shaded part. Inside the wedge there are three equilibria, and the system may arrive either to the lower attractor $\left(P_{1}, \alpha_{1}\right)$ with $\alpha_{1}<\alpha^{*}$ or to the higher attractor $\left(P_{3}, \alpha_{3}\right)$; the latter, for a small part near the cusp point, has $\alpha_{3}<\alpha^{*}$. These two possibilities combine into the shaded part of figure 4 within the wedge (see the inset for finer detail).

If $\alpha^{*}$ is the unique local maximum of $R_{0}$ (as it is the case when $\beta$ is as given in (9)), then the equilibrium density of infected hosts always decreases after introducing the predator. Recall that in absence of the predator, $S(0, \alpha)$ is minimized at $\alpha=\alpha^{*}$. In equation $(4 \mathrm{c}),(\mu+k \alpha+\nu) / \beta(\alpha)$ will thus increase when $\alpha$ evolves away from $\alpha^{*}$, and the positive predator density further increases $S(P, \alpha)$. Because $S+I=N$ is constant, the equilibrium value of $I$ must be less with the predator than without. However, at least in the example shown in figure 4, the combined death rate due to 
the predator and to the disease increases as compared to the predator-free equilibrium (data not shown). The predator can therefore be used as biological control to reduce the incidence of the disease, but this is not necessarily beneficial for the host species.

\subsection{Eco-evolutionary cycles without time-scale separation}

Finally, we return to the full model in equations (1) and relax the assumptions that the host dynamics in (1a)-(1b) is fast and $r \rightarrow \infty$. With finite $r$, we must specify how the birth rate of the host depends on its population density. We assume the host population dynamics to be logistic with $b(N)=1-N / M$, where $M$ is the maximum number of individuals (note that $M$ is greater than the host "carrying capacity", i.e., the equilibrium size of the host population in isolation). The full model is hard to investigate analytically. We integrated equations (1) numerically, using the trade-off functions in (9), and gradually decreased the time scale separation while changed the parameters to retain the cycles until we arrived at the example shown in figure 5. This example demonstrates that eco-evolutionary cycles of predator density and pathogen virulence are possible also without time scale separation, but the method of obtaining this example unfortunately does not let us judge how common the cycles are when the time-scale separation assumptions are relaxed. Exploring the full four-dimensional dynamics in a high-dimensional parameter space is beyond the scope of this paper.

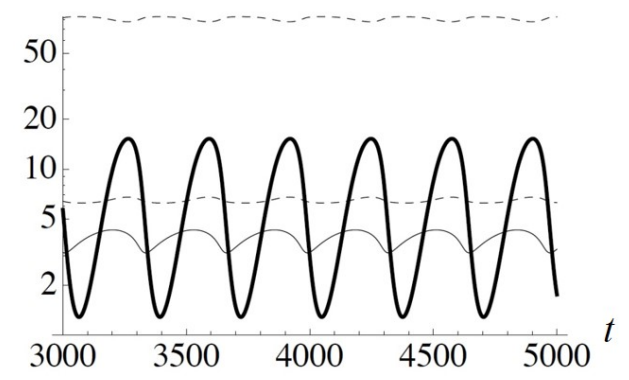

Figure 5: Eco-evolutionary cycles without time-scale separation. Lower dashed line, $S(t)$; upper dashed line, $I(t)$; thick line, $P(t)$; thin line, $\alpha(t)$. Notice the logarithmic vertical scale. Parameters: $r=10, M=$ $100, \mu=1, \nu=5, k=0.01, c=0.01, \gamma=0.1, d=0.3, v=0.35, m=0.03 n, n=0.005, l=1.6$.

\section{Discussion}

\subsection{Evolution of virulence}

We have shown that the virulence of a pathogen can exhibit evolutionary cycles when hosts infected with more virulent pathogens are subject to increased predation and the pathogen evolves at a speed comparable to predator population dynamics. Given that predators are an important cause of mortality, can prefer infected prey (Murray et al. 1997; Johnson et al. 2006), and pathogens evolve quickly compared to the population dynamics of many vertebrate predators (Fenner 1983), the basic assumptions of our model appear to be biologically plausible. 
The key component of the eco-evolutionary cycle is that the pathogen evolves low virulence as a response to high predator density. Introducing an unavoidable factor of extra host mortality selects for an increase of pathogen virulence, because this ensures a higher transmission rate, and the pathogen needs to infect new hosts before its current host dies (Lenski and May 1994). If however the pathogen can avoid the extra mortality of its host by evolving lower virulence, then this may well be an adaptive response (Williams and Day 2001; Donnelly et al. 2012). In our model, predation is such a factor of avoidable mortality, because the predator's capture rate towards the infected depends on virulence such that the pathogen can escape predation via evolving lower virulence. This mechanism was first described by Choo et al. (2003) for a generalist predator with constant population density. The same mechanism drives the cycles in our model assuming a specialist predator with variable density as follows. At high predator density virulence decreases and easy prey disappear, so that predator density gradually decreases; when predator density is low, then virulence increases, so that the infected become easy prey and the predator returns to high density. In contrast, the introduction of the predator always led to higher virulence in the model of Morozov and Adamson (2011), where even though the predator selectively killed the infected hosts, the rate of predation was independent of the virulence of the infecting pathogen.

Most eco-evolutionary models assume that evolution is slow compared to population dynamics. Cycles nevertheless occur in the population dynamics of host-pathogen-predator systems when the predator has a Holling II functional response, and, interestingly, these cycles do not only reflect the well-known limit cycles of the Rosenzweig-MacArtur (1963) model but can persist even if predator density is fixed (Hall et al. 2005; Roy and Holt 2008). Morozov and Adamson (2011) investigated the evolution of virulence assuming slow evolution and population cycles generated via the Holling II functional response. In our model, however, we excluded any purely ecological cycles by assuming a linear functional response and time-scale separation between the host (prey) and the predator. Several types of evolutionary cycles can occur also with slow evolution (Khibnik and Kondrashov 1997). These however require either more than one evolving traits or more than one ecological attractors; and since in our model only virulence evolves and the ecological system has a unique attractor for any fixed virulence, the cycles disappear when the ecological and evolutionary dynamics are decoupled. With slow evolution, we find evolutionary branching of virulence. This agrees with the result of Morozov and Best (2012), who found evolutionary branching in a model similar to ours except that they assumed a sterilizing disease, logistic host population dynamics on the same timescale as the predator, and no recovery. Our finding thus confirms that predation can readily lead to the diversification of pathogens irrespectively of some details of the model.

We investigated the eco-evolutionary cycles assuming that evolutionary change is a speed constant $v$ times the selection gradient (cf. equation (4b)). This assumption is valid when selection operates on a narrow distribution of standing variability (Abrams et al. 1993a; Day and Proulx 2004). An alternative approach is to assume that evolution is mutation-limited and use the canonical equation of adaptive dynamics (Dieckmann and Law 1996; Durinx et al. 2008). With mutation rate $u(\alpha)$ and mutation variance $\sigma^{2}(\alpha)$, the canonical equation in our model reads

$$
\frac{d \alpha}{d t}=\frac{1}{2} u(\alpha) \sigma^{2}(\alpha)(N-S(P, \alpha))\left[\beta^{\prime}(\alpha) S(P, \alpha)-k-f^{\prime}(\alpha) P\right]
$$

i.e., the constant speed factor $v$ in (4b) is replaced with a dynamical variable (note that even if $u$ and $\sigma^{2}$ are constants, the speed factor of the canonical equation contains the variable number 
of infected, $N-S(P, \alpha)$ ). Replacing (4b) with the canonical equation thus leads to different dynamics. This however does not affect the position of the equilibria; in both (4b) and the canonical equation, virulence is at equilibrium when the selection gradient vanishes, and therefore the saddlenode bifurcation lines in figures 2-3 are the same under the canonical equation as for equation (4b). The Jacobian with the canonical equation is the same as the Jacobian given in (6) but with $v=\frac{1}{2} u(\alpha) \sigma^{2}(\alpha)(N-S(P, \alpha))$ evaluated at the equilibrium $(P, \alpha)$, which implies that the Hopf and homoclinic bifurcation lines are different for the two different dynamics. Nevertheless, the construction method of section 3.1 can be used also with the canonical equation to obtain a Bogdanov-Takens point and, by implication, a Hopf bifurcation line; only the proof in Appendix A is not directly applicable to the canonical equation so that the supercriticality of the Hopf bifurcation has to be ascertained separately.

\subsection{A comparison to predator-prey models}

Eco-evolutionary cycles are extremely well studied in predator-prey models (see Abrams 2000b for a review). Of particular interest here are models where, as in our case, the nonequilibrium dynamics depends on the interaction between population and evolutionary dynamics, such that the cycles disappear in the limit of slow evolution. This happens, for example, when the predator's costly capture rate evolves in a predator-prey system with a nonlinear numerical response of the predator (Abrams 1992), or when the prey's vulnerability to the predator evolves under a Holling II functional response of the predator (Abrams and Matsuda 1997a; Mougi 2012). The model of Abrams (2003) is particularly similar to ours. This model considers the limiting resource of the prey explicity, and assumes that prey searching for the resource more actively are also more vulnerable to predation. Both prey and predator have linear functional responses, and although Abrams (2003) considers also nonlinear numerical responses for the prey, his example uses a linear response. If we think, in our model, of the susceptible hosts as "resource" (i.e., for the pathogen) and the infected hosts as "prey" (which consumes the "resource", in our case by infecting it), then our equations (1) bear strong similarities to equations (1a-d) of Abrams (2003). The evolving trait in Abrams' model, the search activity of prey, is the constant of proportionality in the mass action term that tranfers resource into prey; this is directly analogous to the transmission rate of the infection in our model. Abrams assumes that the search activity is traded off with the background mortality of the prey and with the capture rate of the predator. With an invertible transmission-virulence trade-off, it is an easy exercise to rewrite our trade-off functions such that the transmission rate is the independent variable; and with this substitution, three of the four equations of the two models are reduced to the same form. The models differ in the dynamics of the resource $v s$ susceptibles. To recover also the first equation of Abrams (2003), we would need to assume no recovery, no predation on susceptibles, a sterilizing disease, and (least realistically) that the per capita birth rate of hosts depends on the density of only the susceptibles but not of the infected.

Comparing the Jacobian matrix of our model given in (6) with the condition for a fitness minimum in (10) reveals that if (10) holds at an equilibrium and the speed constant $v$ is sufficiently large, then the trace of the Jacobian is positive, i.e., the equilibrium of the ODE system in (4) is not stable. This link between fitness minima and nonequilibrium dynamics has been discovered in predator-prey systems (Abrams and Matsuda 1997b; Abrams 2003), and is particularly relevant for the study of fast-evolving pathogens. The general conditions under which this link exists have 
however not yet been given.

The rich literature on the nonequilibrium dynamics of predator-prey models inspires a number of questions for future research, especially concerning the dynamics and evolution of the predator. In the present paper, we emphasised that predators are an important source of mortality for diseased hosts; but conversely, diseased hosts are important resources for predators, and therefore the dynamics of the disease shapes the dynamics and evolution of predators.

First, it is well known that when a predator-prey system settles at a limit cycle, more predators can coexist than the number of prey species, provided that the predators have sufficiently different nonlinearities in their functional response (Hsu et al. 1978; Armstrong and McGehee 1980; Abrams et al. 2003; Abrams 2006). A predator with (nearly) linear functional response reacts to the time-average of prey density, whereas a predator with a strongly nonlinear functional response is at an advantage when prey density is low. Could predators coexist also in our model if they have different functions linking virulence to the capture rate $(f(\alpha))$, such that one reacts to the average value of $\alpha I$ whereas the other is at an advantage when virulence is low?

Second, what are the consequences of increasing the predator's mortality e.g. by harvesting? Counterintuitively, the density of predators exploiting structured prey populations may increase with increasing mortality (Abrams and Quince 2005), a phenomenon called the "hydra effect" (see Abrams 2009a for a review). In our model, the prey is structured according to being healthy or infected. With the timescale separation assumption made in this paper, predator density decreases with harvesting when the pathogen does not evolve; it is however unclear what happens when virulence changes and how the eco-evolutionary cycles change when the predator is harvested. If the pathogen evolves higher virulence as a response to decreasing predator density (i.e., if (8) holds), will this slow down the decline of the predator by providing easier prey to capture (cf. Abrams 2009b)?

Third, how should the predator evolve its ability to capture the prey? Under which conditions should the predator invest into improving its capture rate towards healthy prey, or towards prey infected but with low-virulence strains of the pathogen? Abrams $(1986,1997)$ investigated the evolutionary responses of predators to changing prey traits and vice versa for unstructured prey; how do the coevolutionary patterns change in the presence of a fast-evolving pathogen?

\subsection{Construction of trade-offs to obtain eco-evolutionary cycles}

We have demonstrated the eco-evolutionary cycles of pathogen virulence and predator density in a concrete example with simple trade-off functions given in (9). Obtaining these cycles is however not surprising in the light of the first part of this paper, where we show that one can actually choose the trade-offs such that the existence of stable limit cycles is guaranteed. Although technically more involved, this construction method is based on the same idea as critical function analysis, a technique used to construct trade-offs that yield evolutionary branching (de Mazancourt and Dieckmann 2004; Bowers et al. 2005; Kisdi 2006; Geritz et al. 2007). Critical function analysis can establish or disprove that a certain ecological model is able to facilitate the evolution of diversity via gradual divergence (see Svennungsen and Kisdi 2009; Boldin et al. 2009; Best et al. 2010; 
Morozov and Best 2012 for applications to virulence evolution and Kisdi and Boldin 2013 for a related construction method for incidence functions). Non-equilibrium evolutionary dynamics is another important and intriguing process driven by ecological interactions. Our paper is a first attempt to provide a similar construction method also for nonequilibrium evolutionary dynamics.

Using a constructive method will prove by example that a given outcome (such as evolutionary branching or evolutionary cycles) is possible in a given ecological system. Constructive methods eliminate the ad hoc element of choosing a simple formula to specify a trade-off function, and give conditions directly in terms of local properties such as slopes and convexities of the trade-off functions. The resulting examples have however no predictive power; since the trade-off functions have been constructed specifically to arrive at a given outcome, they do not inform how readily this outcome may be expected. In the case of critical function analysis, one can give intervals of tradeoff convexities that lead to evolutionary branching (de Mazancourt and Dieckmann 2004), and the width of this interval gives some information about how likely branching is. A similar extension is however not straightforward for the construction method we used in this paper. The reason is that limit cycles can undergo global bifurcations (such as the homoclinic bifurcation in figures 2-3) or bifurcations otherwise less tractable (such as a fold bifurcation of limit cycles, Kuznetsov 2004, p. 170). Constructing trade-offs with a supercritical Hopf bifurcation ensures that stable limit cycles occur in the vicinity, but finding the precise set of trade-offs where the cycle persists is much harder because the cycle disappears e.g. via a homoclinic bifurcation. The lack of predictive power prompted us to carry out also a traditional bifurcation analysis with a specific family of trade-offs, presented in the second part of the paper (figures 2-3). However, the mere fact that properly chosen trade-offs lead to eco-evolutionary cycles whereas other trade-offs lead to stable equilibria highlights that the trade-offs are essential components of the model, and cautions that specifying the trade-off functions in an ad hoc manner introduces an unjustifiable element of accidentality.

\section{Acknowledgements}

Comments of two anonymous reviewers helped to improve the presentation. This research was financially supported by the Academy of Finland and by the Slovenian Research Agency (project no. BI-FI/12-13-010).

\section{References}

Abrams P. A. 1986. Adaptive responses of predators to prey and prey to predators: The failure of the arms-race analogy. Evolution 40: 1229-1247.

Abrams P. A. 1992. Adaptive foraging by predators as a cause of predator-prey cycles. Evol. Ecol. 6: $56-72$.

Abrams P. A. 1997. Evolutionary responses of foraging-related traits in unstable predator-prey systems. Evol. Ecol. 11: 673-686.

Abrams P. A. 2000a. Character shifts of prey species that share predators. Am. Nat. 156: S45-S61. 
Abrams P. A. 2000b. The evolution of predator-prey interactions: Theory and evidence. Annu. Rev. Ecol. Syst. 31: 79-105.

Abrams P. A. 2003. Can adaptive evolution or behaviour lead to diversification of traits determining a trade-off between foraging gain and predation risk? Evol. Ecol. Res. 5: 653-670.

Abrams P. A. 2006. Adaptive change in the resource-exploitation traits of a generalist consumer: The evolution and coexistence of generalists and specialists. Evolution 60: 427-439.

Abrams P. A. 2009a. When does greater mortality increase population size? The long history and diverse mechanisms underlying the hydra effect. Ecol. Lett. 12: 462-474.

Abrams P. A. 2009b. Adaptive changes in prey vulnerability shape the response of predator populations to mortality. J. theor. Biol. 261: 294-304.

Abrams P. A. 2012. The eco-evolutionary responses of a generalist consumer to resource competition. Evolution 66: 3130-3143.

Abrams P. A., C. E. Brassil \& R. D. Holt. 2003. Dynamics and responses to mortality rates of competing predators undergoing predator-prey cycles. Theor. Pop. Biol. 64: 163-176.

Abrams P. A., Y. Harada \& H. Matsuda. 1993a. On the relationship between quantitative genetic and ESS models. Evolution 47: 982-985.

Abrams P. A. \& H. Matsuda. 1997a. Prey adaptation as a cause of predator-prey cycles. Evolution 51: $1742-1750$.

Abrams P. A. \& H. Matsuda. 1997b. Fitness minimization and dynamic instability as a consequence of predator-prey coevolution. Evol. Ecol. 11: 1-20.

Abrams P. A., H. Matsuda \& Y. Harada. 1993b. Evolutionarily unstable fitness maxima and stable fitness minima of continuous traits. Evol. Ecol. 7: 465-487.

Abrams P. A. C. Quince. 2005. The impact of mortality on predator population size and stability in systems with stage-structured prey. Theor. Pop. Biol. 68: 253-266.

Anderson R. M. \& R. M. May. 1986. The invasion, persistence and spread of infectious diseases within animal and plant communities. Phil. Trans. R. Soc. Lond. B 314: 533-570.

Andreasen V. \& A. Pugliese. 1995. Pathogen coexistence induced by density-dependent host mortality. J. theor. Biol. 177: 159-165.

Armstrong R.A., R. McGehee. 1980. Competitive exclusion. Am. Nat. 115:151-170.

Best A., A. White, E. Kisdi, J. Antonovics, M. A. Brockhurst \& M. Boots. 2010. The evolution of 
host-parasite range. Am. Nat. 176: 63-71.

Boldin B., S. A. H. Geritz \& E. Kisdi. 2009. Superinfections and adaptive dynamics of pathogen virulence revisited: A critical function analysis. Evol. Ecol. Res. 11: 153-175.

Bowers R. G., A. Hoyle, A. White \& M. Boots. 2005. The geometric theory of adaptive evolution: Trade-off and invasion plots. J. theor. Biol. 233: 363-377.

Bremermann H. J. \& H. R. Thieme. 1989. A competitive exclusion principle for pathogen virulence. J. Math. Biol. 27: 179-190.

Choo K., P. D. Williams \& T. Day. 2003. Host mortality, predation and the evolution of parasite virulence. Ecol. Lett. 6: 310-315.

Day T. \& S. R. Proulx. 2004. A general theory for the evolutionary dynamics of virulence. Am. Nat. 163: E40-E63.

de Mazancourt C. \& U. Dieckmann. 2004. Trade-off geometries and frequency-dependent selection. Am. Nat. 164: 765-778.

Dieckmann U. \& R. Law. 1996. The dynamical theory of coevolution: A derivation from stochastic ecological processes. J. Math. Biol. 34: 579-612.

Dieckmann U. \& J. A. J. Metz. 2006. Surprising evolutionary predictions from enhanced ecological realism. Theor. Pop. Biol. 69: 263-281.

Donnelly R., A. Best, A. White \& M. Boots. 2012. Seasonality selects for more acutely virulent parasites when virulence is density dependent. Proc. R. Soc. B 280: 20122464.

Durinx M., J. A. J. Metz \& G. Meszena. 2008. Adaptive dynamics for physiologically structured population models. J. Math. Biol. 56: 673-742.

Fenner F. 1983. The Florey Lecture, 1983: Biological control, as exemplified by smallpox eradication and myxomatosis. Proc. R. Soc. Lond. B 218: 259-285.

Geritz S. A. H., E. Kisdi, G. Meszéna \& J. A. J. Metz. 1998. Evolutionarily singular strategies and the adaptive growth and branching of the evolutionary tree. Evol. Ecol. 12: 35-57.

Geritz S. A. H., E. Kisdi \& P. Yan. 2007. Evolutionary branching and long-term coexistence of cycling predators: Critical function analysis. Theor. Pop. Biol. 71: 424-435.

Gyllenberg M., X. Liu \& P. Yan. 2012. An eco-epidemiological model in two competing species. Differ. Equ. Appl. 4: 495-519.

Hall S. R., M. A. Duffy \& C. E. Caceres. 2005. Selective predation and productivity jointly drive 
complex behavior in host-parasite systems. Am. Nat. 165: 70-81.

Hatcher M. J., J. T. A. Dick \& A. M. Dunn. 2006. How parasites affect interactions between competitors and predators. Ecol. Lett. 9: 1253-1271.

Hethcote H. W., W. Wang, L. Han \& Z. Ma. 2004. A predator-prey model with infected prey. Theor. Pop. Biol. 66: 259-268.

Hilker F. M. \& K. Schmitz. 2008. Disease-induced stabilization of predatorprey oscillations. J. theor. Biol. 255: 299-306.

Hoyle A., A. Best \& R. G. Bowers. 2012. Evolution of host resistance towards pathogen exclusion: The role of predators. Evol. Ecol. Res. 14: 125-146.

Hsu S. B., S. P. Hubbell \& P. Waltman. 1978. Competing predators. SIAM J. Appl. Math. 35: 617-625.

Hudson P. J., A. P. Dobson \& D. Newborn. 1992. Do parasites make prey vulnerable to predation? Red grouse and parasites. J. Anim. Ecol. 61: 681-692.

Johnson P. T. J., D. E. Stanton, E. R. Preu, K. J. Forshay \& S. R. Carpenter. 2006. Dining on disease: How interactions between infection and environment affect predation risk. Ecology 87: 1973-1980.

Khibnik A. I. \& A. S. Kondrashov. 1997. Three mechanisms of Red Queen dynamics. Proc. R. Soc. Lond. B 264: 1049-1056.

Kisdi E. 2006. Trade-off geometries and the adaptive dynamics of two coevolving species. Evol. Ecol. Res. 8: 959-973.

Kisdi E. \& B. Boldin. 2013. A construction method to study the role of incidence in the adaptive dynamics of pathogens with direct and environmental transmission. J. Math. Biol. 66: 10211044.

Kooi B. W., G. A. K. van Voorn \& K. P. Das. 2011. Stabilization and complex dynamics in a predator-prey model with predator suffering from an infectious disease. Ecol. Complexity 8: 113122.

Kuznetsov Yu. 2004. Elements of applied bifurcation theory. Springer Verlag.

Lenski R. \& R. M. May. 1994. The evolution of virulence in parasites and pathogens: Reconciliation between two competing hypotheses. J. theor. Biol. 169: 253-265.

Maynard Smith J. 1982. Evolution and the theory of games. Cambridge University Press, Cambridge. 
Metz J. A. J., S. D. Mylius \& O. Diekmann. 2008. When does evolution optimize? Evol. Ecol. Res. 10: 629-654.

Morozov A. Yu. \& M. W. Adamson. 2011. Evolution of virulence driven by predator-prey interaction: Possible consequences for population dynamics. J. theor. Biol. 276: 181-191.

Morozov A. \& A. Best. 2012. Predation on infected host promotes evolutionary branching of virulence and pathogens' biodiversity. J. theor. Biol. 307: 29-36.

Mougi A. 2012. Predator-prey coevolution driven by size selective predation can cause antisynchronized and cryptic population dynamics. Theor. Pop. Biol. 81: 113-118.

Murray D. L., J. R. Cary \& L. B. Keith. 1997. Interactive effects of sublethal nematodes and nutritional status on snowshoe hare. J. Anim. Ecol. 66: 250-264.

Otti O., I. Ganterbein-Ritter, A. Jacot \& M. W. G. Brinkhof. 2012. Immune response increases predation risk. Evolution 66: 732-739.

Packer C., R. D. Holt, P. J. Hudson, K. D. Lafferty \& A. P. Dobson. 2003. Keeping the herds healthy and alert: Implications of predator control for infectious disease. Ecol. Lett. 6: 797-802.

Peng G. \& Y. Jiang. 2011. Practical computation of normal forms of the Bogdanov-Takens bifurcation. Nonlinear Dyn. 66: 99-132.

Roberts M. G. \& J. A. P. Heesterbeek. 2013. Characterizing the next-generation matrix and basic reproduction number in ecological epidemiology. J. Math. Biol. 66: 1045-1064.

Rosenzweig M. L. \& R. H. MacArthur. 1963. Graphical representation and stability conditions of predator-prey interactions. Am. Nat. 97: 209-223.

Roy M. \& R. D. Holt. 2008. Effects of predation on host-pathogen dynamics in SIR models. Theor. Pop. Biol. 73: 319-331.

Strauss S. Y., J. A. Lau \& S. P. Carroll. 2006. Evolutionary responses of natives to introduced species: What do introductions tell us about natural communities? Ecol. Lett. 9: 357-374.

Svennungsen T. O. \& E. Kisdi. 2009. Evolutionary branching of virulence in a single infection model. J. theor. Biol. 257: 408-418.

Venturino E. 2001. The effects of diseases on competing species. Mat. Biosci. 174: 111-131.

Venturino E. 2002. Epidemics in predator-prey models: disease in the predators. IMA J. Math. Appl. Med. Biol. 19: 185-205.

Venturino E. 2007. How diseases affect symbiotic communities. Math. Biosci. 206: 11-30. 
Williams P. D. \& T. Day. 2001. Interactions between sources of mortality and the evolution of parasite virulence. Proc. R. Soc. Lond. B 268: 2331-2337.

\section{Appendix A}

In the main text, we have shown how $\beta^{\prime}(\alpha), \beta^{\prime \prime}(\alpha)$ and $f^{\prime}(\alpha)$ can be chosen such that given the values of $\alpha, \beta(\alpha)$ and $f(\alpha)$, the system in (4) has an equilibrium with two zero eigenvalues of the Jacobian. What remains to be seen is that the nondegeneracy condition of the Bogdanov-Takens bifurcation is satisfied, and moreover that the Hopf bifurcation associated with the Bogdanov-Takens point is supercritical rather than subcritical (see Kuznetsov 2004). In terms of the coefficients $a, b$ of the critical normal form

$$
\begin{aligned}
& \dot{x}_{1}=x_{2} \\
& \dot{x}_{2}=a x_{1}^{2}+b x_{1} x_{2},
\end{aligned}
$$

the nondegeneracy condition is $a b \neq 0$ and the Hopf bifurcation is supercritical if $a b<0$. In this Appendix, we show that the so far free convexity $f^{\prime \prime}(\alpha)$ can be chosen such that $a b<0$ is satisfied provided that $a$ and $b$ do not vanish simultaneously; and we also show that the latter condition holds when certain special values of the third derivatives of the trade-off functions are avoided.

Let $g(P, \alpha)$ and $h(P, \alpha)$ be the right hand sides of (4a) and (4b), respectively, and let $p_{i j}$ and $q_{i j}$ denote their Taylor coefficients according to

$$
p_{i j}=\frac{1}{i ! j !} \frac{\partial^{i+j} g}{\partial P^{i} \partial \alpha^{j}}
$$

and

$$
q_{i j}=\frac{1}{i ! j !} \frac{\partial^{i+j} h}{\partial P^{i} \partial \alpha^{j}}
$$

where the derivatives are evaluated at the bifurcation point (note that the first derivatives are the elements of the Jacobian, i.e., $p_{10}=J_{11}, p_{01}=J_{12}, q_{10}=J_{21}$ and $q_{01}=J_{22}$ ). The coefficients $a$ and $b$ are then given by

$$
\begin{aligned}
a & =\frac{p_{10}^{3}}{p_{01}^{2}} p_{02}-\frac{p_{10}^{2}}{p_{01}}\left(p_{11}-q_{02}\right)+p_{10}\left(p_{20}-q_{11}\right)+p_{01} q_{20} \\
b & =-\frac{p_{10}}{p_{01}}\left(p_{11}+2 q_{02}\right)+2 p_{20}+q_{11}
\end{aligned}
$$

(Peng and Jiang 2011, equation (17)).

The technical calculations necessary for the following have been done using Mathematica 6.0 (Wolfram Research). Evaluating the Taylor coefficients in (13a,b) at the bifurcation point amounts to substituting $P, \beta^{\prime}(\alpha), \beta^{\prime \prime}(\alpha)$ and $f^{\prime}(\alpha)$ as obtained in the main text. After this substitution, the Taylor coefficients depend on $f^{\prime \prime}(\alpha)$; moreover, $q_{02}$ depends also on the third derivatives of the trade-off functions $\beta^{\prime \prime \prime}(\alpha)$ and $f^{\prime \prime \prime}(\alpha)$ (other Taylor coefficients do not). $p_{i j}$ and $q_{i j}$ are too complicated to show here, but we shall use only the following properties: 
(i) $p_{10}, p_{01}, p_{20}, p_{11}, q_{20}$ and $q_{02}$ do not depend on $f^{\prime \prime}(\alpha)$;

(ii) $q_{02}$ can be written as $q_{02}=Q+\eta \beta^{\prime \prime \prime}(\alpha)+\theta f^{\prime \prime \prime}(\alpha)$, where $Q, \eta, \theta$ are numbers and $\eta>0$ whereas $\theta<0$;

(iii) $p_{10}=J_{11}<0$ (cf. (6));

(iv) $p_{01}=J_{12}>0\left(\right.$ cf. (6) and $\left.f^{\prime}(\alpha)>0\right)$;

(v) $q_{20}=0$;

(vi) $p_{02}$ can be written as $p_{02}=C_{02}+\kappa_{02} f^{\prime \prime}(\alpha)$, where $C_{02}$ is a number and $\kappa_{02}=\frac{(d-\gamma c N) P}{2 f(\alpha)}$ is positive;

(vii) $q_{11}$ can be written as $q_{11}=C_{11}+\kappa_{11} f^{\prime \prime}(\alpha)$, where $C_{11}$ is a number and $\kappa_{11}=-v \gamma \frac{f(\alpha)}{\beta(\alpha)} \frac{\mu+k \alpha+\nu}{\gamma(c+f(\alpha)) N-d}$ is negative.

First notice that $b$ depends on $f^{\prime \prime}(\alpha)$ only through $q_{11} . b$ is therefore linear in $f^{\prime \prime}(\alpha)$ with the nonzero coefficient $\kappa_{11}$, and $b$ changes sign at $f^{\prime \prime}(\alpha)=f_{c}^{\prime \prime}$ where

$$
f_{c}^{\prime \prime}=\frac{1}{\kappa_{11}}\left[\frac{p_{10}}{p_{01}}\left(p_{11}+2 q_{02}\right)-2 p_{20}-C_{11}\right]
$$

$f^{\prime \prime}(\alpha)$ can therefore be chosen such that $a b<0$, provided that $a$ does not vanish at $f^{\prime \prime}(\alpha)=f_{c}^{\prime \prime}$. Substituting $f^{\prime \prime}(\alpha)=f_{c}^{\prime \prime}$ into $a$ as given in (14a) and using properties (v)-(vi) yields the expression

$$
\begin{aligned}
& \frac{p_{10}^{3}}{p_{01}^{2}}\left[C_{02}+\frac{\kappa_{02}}{\kappa_{11}}\left(\frac{p_{10}}{p_{01}}\left(p_{11}+2 q_{02}\right)-2 p_{20}-C_{11}\right)\right]-\frac{p_{10}^{2}}{p_{01}}\left(p_{11}-q_{02}\right)+ \\
& +p_{10}\left[3 p_{20}-\frac{p_{10}}{p_{01}}\left(p_{11}+2 q_{02}\right)\right]
\end{aligned}
$$

Next to numbers, this expression contains the third derivatives $\beta^{\prime \prime \prime}(\alpha)$ and $f^{\prime \prime \prime}(\alpha)$ via $q_{02}$. The coefficient of $q_{02}$ in (16) simplifies to

$$
\frac{p_{10}^{2}}{p_{01}}\left(2 \frac{p_{10}^{2}}{p_{01}^{2}} \frac{\kappa_{02}}{\kappa_{11}}-1\right)<0
$$

By (17) and property (ii) above, (16) is a linear function of the third derivatives $\beta^{\prime \prime \prime}(\alpha)$ and $f^{\prime \prime \prime}(\alpha)$ with nonzero coefficients. (16) therefore does not vanish except for a set of values $\left\{\beta^{\prime \prime \prime}(\alpha), f^{\prime \prime \prime}(\alpha)\right\}$ of zero measure. Should (16) vanish, one can unfold this degeneracy by changing either $\beta^{\prime \prime \prime}(\alpha)$ or $f^{\prime \prime \prime}(\alpha)$.

This completes the proof that $f^{\prime \prime}(\alpha)$ can be chosen such that $a b<0$. In practice, when the parameter values of the model are specified and the values for $\alpha, \beta(\alpha)$ and $f(\alpha)$ are chosen, $\beta^{\prime}(\alpha)$ and $f^{\prime}(\alpha)$ evaluate to numbers whereas $\beta^{\prime \prime}(\alpha)$ is a simple linear function of $f^{\prime \prime}(\alpha)$ (see main text). One can choose numerical values also for $\beta^{\prime \prime \prime}(\alpha)$ and $f^{\prime \prime \prime}(\alpha)$ such that $f^{\prime \prime}(\alpha)$ is the only non-numerical element in $a$ and $b$. The product $a b$ can then be evaluated numerically as a function of $f^{\prime \prime}(\alpha)$, and a value of $f^{\prime \prime}(\alpha)$ can be chosen where $a b<0$. 
We note that $a b$ is quadratic in $f^{\prime \prime}(\alpha)$ and the coefficient of the quadratic term is $p_{10} \kappa_{11}\left[\left(\frac{p_{10}}{p_{01}}\right)^{2} \kappa_{02}-\right.$ $\left.\kappa_{11}\right]>0$. Hence choosing either sufficiently large or sufficiently small values of $f^{\prime \prime}(\alpha)$ will make $a b$ positive, and therefore will lead to a model with an unstable limit cycle between the Hopf and homoclinic bifurcation lines. Such a model does not exhibit stable cycles, but does have an interesting type of bistability. Further, by changing $f^{\prime \prime}(\alpha)$ one can also find a codimension 3 bifurcation where $a b=0 . a b$ is also quadratic in $q_{02}$ with coefficient $-2 \frac{p_{10}^{3}}{p_{01}^{2}}>0$. Large values of $\beta^{\prime \prime \prime}(\alpha)$ with small (large negative) values of $f^{\prime \prime \prime}(\alpha)$ will therefore make $a b$ positive for any given $f^{\prime \prime}(\alpha)$, and the same happens for small (large negative) values of $\beta^{\prime \prime \prime}(\alpha)$ with large values of $f^{\prime \prime \prime}(\alpha)$. With the possibility of both stable and unstable limit cycles and a codimension 3 bifurcation, the model exhibits very rich dynamics depending on the trade-off functions.

\section{Appendix B}

In this Appendix, we investigate the existence and stability of boundary equilibria of the model in (4) with trade-offs as given in (9). In the predator-free system, the pathogen evolves to an equilibrium $\alpha^{*}$ of $(4 \mathrm{~b})$ with $P=0$. With $k>0$ and any saturating $\beta$ function, virulence remains bounded and hence $\alpha^{*}$ exists. When $\beta(0)=0$ (as in $(9 \mathrm{a})$ ), pathogens of too low virulence are not viable and therefore the optimal virulence is positive. The optimal virulence is given implicitly by

$$
\beta^{\prime}\left(\alpha^{*}\right)=\frac{k \beta\left(\alpha^{*}\right)}{\mu+k \alpha^{*}+\nu}
$$

For $\alpha^{*}$ to be an optimum, or equivalently to be a stable equilibrium of $(4 \mathrm{~b}), \beta^{\prime \prime}\left(\alpha^{*}\right)$ needs to be negative; it is always so and $\alpha^{*}$ is unique when the trade-off function is everywhere concave as in (9a).

When introduced at a low density, the predator can invade the predator-free system if its initial growth rate is positive, i.e., if

$$
f\left(\alpha^{*}\right)>\frac{d / \gamma-c N}{N-\frac{\mu+k \alpha^{*}+\nu}{\beta\left(\alpha^{*}\right)}}
$$

Since for all $\alpha>0, f(\alpha)$ in (9b) increases with the value of parameter $l$ and also increases with increasing $n$ and $m$ proportionally (recall that $m / n$ is kept constant), the predator is able to invade when $l$ or $n$ is sufficiently large, provided that the saturation value $m / n$ exceeds the right hand side of inequality (19).

By the assumption $\gamma c N<d$, the predator dies out in absence of the pathogen, and therefore the predator cannot drive the pathogen extinct. It follows that there is no boundary equilibrium with no infected hosts but positive predator density. There is also no boundary equilibrium without a positive density of susceptibles, since in absence of susceptibles the density of infected decreases. Of the remaining boundary equilibria, $(S, I, P, \alpha)=\left(N, 0,0, \alpha^{*}\right)$ is always unstable by the assumption that $\alpha^{*}$ is viable, and $\left(S\left(0, \alpha^{*}\right), N-S\left(0, \alpha^{*}\right), 0, \alpha^{*}\right)$ is unstable provided that (19) holds. This last boundary equilibrium is the only one where a transcritical bifurcation can occur. 